\title{
APRENDIZADO DE MÁQUINA APLICADO PARA AUXÍLIO AO MOTORISTA UTILIZANDO RASPBERRY PI
}

\author{
MACHINE LEARNING APPLIED FOR AID TO DRIVER USING RASPBERRY \\ PI
}

Luan Lourenço Esteves ${ }^{1}$, Francisco Assis da Silva ${ }^{1}$, Leandro Luiz de Almeida ${ }^{1}$, Danillo Roberto Pereira ${ }^{1}$, Mário Augusto Pazoti ${ }^{1}$, Almir Olivette Artero ${ }^{2}$

\section{${ }^{1}$ Universidade do Oeste Paulista}

Faculdade de Informática de Presidente Prudente

E-mail: luan_esteves@live.com, \{chico, Ilalmeida, danilopereira, mario\}@unoeste.br,

\author{
${ }^{2}$ Unesp - Universidade Estadual Paulista \\ E-mail: almir@fct.unesp.br
}

RESUMO - O Brasil tem a quinta maior taxa de mortes no trânsito do planeta. Geralmente os acidentes são causados por falha humana, envolvendo desatenção e desrespeito à legislação. A fim de auxiliar o motorista a agir de forma preventiva e responsável, sistemas computacionais podem estabelecer meios para emitir alertas ao reconhecer situações de risco à segurança no trânsito. O desafio apresentado neste trabalho foi realizar a detecção e o reconhecimento de alguns sinais de trânsito considerados necessários à segurança viária. Este trabalho objetivou o desenvolvimento de um sistema embarcado de auxílio ao motorista baseado em visão computacional e aprendizado de máquina. A função do sistema é reconhecer situações perigosas e alertar o motorista a respeito das sinalizações encontradas nas vias (placas de sinais de velocidade máxima permitida, parada obrigatória, preferência e faixas rolamento). Foi utilizado um Raspberry Pi 3 e uma câmera de 5 megapixels para ser o hardware embarcado. $O$ trabalho buscou o desenvolvimento de algoritmos que realizem a tarefa de auxiliar a percepção humana ao guiar veículos, com execução em hardware de baixo processamento em tempo real.

Palavras-chave: Auxílio ao motorista; Aprendizado de Máquina; Visão computacional, Inteligência Artificial.

\footnotetext{
ABSTRACT - Brazil has the fifth highest death toll in the planet. Generally accidents are caused by human failure, involving inattention and disrespect to the law. In order to help the driver to act in a preventive and responsible manner, computer systems can establish
} 
ways to issue alerts when recognizing situations of risk to the safety in the traffic. The challenge of this work was to perform the detection and recognition of some traffic signals considered necessary for road safety. This work aimed at the development of an embedded system of assistance to the driver based on computer vision and machine learning. The function of the system is to recognize dangerous situations and alert the driver to the signals found on the tracks (maximum permissible speed, stop, preference and bearing tracks). We used a Raspberry Pi 3 and a camera of 5 megapixels to be the embedded hardware. The work aimed the development of algorithms that perform the task of assisting human perception in guiding vehicles, with execution in low-processing hardware in real time.

Keywords: Driver's Aid; Machine Learning; Computer Vision; Artificial Intelligence. 


\section{INTRODUÇÃO}

De acordo com a CNT (Confederação Nacional do Transporte), o Brasil possui 1,7 milhões de quilômetros de malha rodoviária, responsáveis por interligar todas as partes do país. Essa infraestrutura suporta quase 100 milhões de veículos em circulação, o que resulta em sobrecarga e aumenta os riscos de acidentes (CNT, 2018).

Nos últimos anos, a tecnologia tem contribuído significativamente com melhorias na questão de segurança no trânsito. Câmeras e sensores são embarcados nos veículos para monitorar o ambiente e possibilitar que sistemas inteligentes auxiliem o condutor. Alguns sistemas ajustam automaticamente a velocidade do veículo levando em consideração o automóvel à frente, enquanto outros realizam aviso de saída de pista e frenagens de emergência. Utilizar a tecnologia a favor do motorista é um recurso inteligente em vista das estatísticas negativas em relação a acidentes de trânsito.

Segundo o Ministério da Saúde, no ano de 2016 foram registrados 37.345 óbitos causados pelos acidentes de trânsito (Sistema de Informações de Mortalidade do Ministério da Saúde, 2016). Em 2017, o DPVAT realizou o pagamento de 284.191 indenizações por invalidez permanente e 41.151 por morte (Relatório Anual DPVAT, 2017).

Segundo o Observatório Nacional de Segurança Viária, $90 \%$ dos acidentes de trânsito são causados por falha humana, envolvendo desatenção e desrespeito à legislação. Dirigir acima da velocidade permitida ou cansado são exemplos de comportamentos que geram situações perigosas no trânsito. Evitar tais situações são, de fato, decisivas para a redução das estatísticas de acidentes (ONSV, 2015).

Para auxiliar motoristas de veículos a agirem de forma preventiva e responsável, sistemas baseados em computação podem estabelecer meios para emitir alertas durante o percurso nas vias. Pesquisas na área de visão computacional e aprendizado e máquina apresentam sistemas para essa finalidade.

Este trabalho contribui com 0 desenvolvimento de um sistema embarcado de baixo custo para emitir alertas durante o percurso nas vias, com propósito de reconhecer a sinalização de velocidade máxima permitida, dê a preferência, parada obrigatória e faixas de rolamento. Os resultados apresentados envolvem a identificação de algumas sinalizações de trânsito e emissão de alertas ao motorista para que contribua com a tomada de decisões do motorista.

As demais seções deste trabalho estão organizadas da seguinte maneira: na Seção 2 são apresentados alguns trabalhos relacionados; na Seção 3 são apresentadas a metodologia e o desenvolvimento do sistema embarcado; na Seção 4 são apresentados os experimentos realizados e os resultados obtidos; por fim, a Seção 6 apresenta as conclusões.

\section{TRABALHOS RELACIONADOS}

Segundo Visvikis et al. (2008), o Laboratório de Pesquisa em Transporte explica que existem dois tipos de sistemas de alerta de alteração de pista. O primeiro alerta o motorista em ocasiões de mudança de pista que podem resultar em colisão (LCA Lane Change Assistance). O segundo informa o motorista quando uma alteração de pista não intencional irá ocorrer (LDW - Lane Departure Warning). Ambos sistemas devem monitorar o tipo de marcação horizontal na pista, identificando locais de ultrapassagem permitida e proibida.

Nan et al. (2016) apresentaram um método para detectar os limites da pista em vídeo. O modelo segue algumas etapas, iniciando com a segmentação da imagem para obter segmentos de linhas precisos em várias cenas de tráfego. Após esta etapa, a imagem ainda apresenta segmentos que não são de fato parte da delimitação da via, necessitando utilizar abordagens para corrigir 
ruídos. É proposta a utilização de um ponto de fuga, onde todas as linhas da pista convergem, e uma estrutura de triângulo, abrangendo todas as faixas de rolamento. Todos os pontos, linhas que não estão presentes dentro da delimitação do ponto de fuga e triângulo, são ruídos e devem ser removidos. Para delimitar as linhas da pista foi utilizada uma máquina de estados, identificando se o estado atual é para esquerda, direita ou linha reta. Segundo os autores, o método é adequado para algumas aplicações em tempo real e foi testado em diferentes tipos de ambientes, condições meteorológicas e iluminação. Algumas imagens com resultados são apresentadas na Figura 1.

Figura 1. Identificação de Faixas de Rolamento.

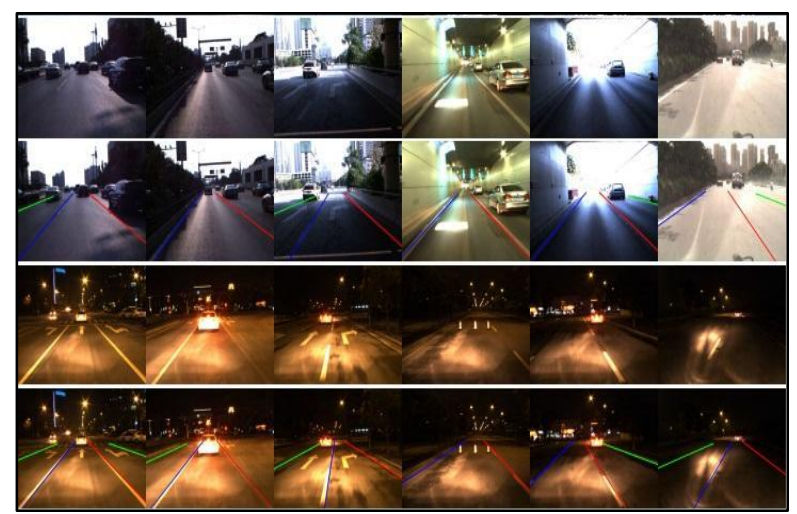

Fonte: (Nan et al, 2016).

Andrade (2017) apresentou um sistema de baixo custo baseado em visão computacional para realizar extração de características e realizar o rastreamento das faixas de rolamento mais próximas do veículo em rodovias. São utilizadas três etapas de processamento (baixo, médio e alto nível) em imagens coletadas a partir de uma câmera monocular instalada na parte central do para-brisa do veículo. A etapa de nível baixo possui como objetivo selecionar uma região de interesse na imagem com altura de 120 linhas, definido com base no tempo de resposta do motorista ao realizar uma frenagem. Essa imagem é utilizada com entrada para o próximo nível (médio), onde é aplicado o detector de bordas de Canny
(Gonzalez; Woods, 2010). Também, é aplicada uma filtragem baseada na orientação do gradiente da imagem com base em um intervalo de ângulos previstos para as linhas de pista. Para reduzir ruídos, foi utilizada uma dilatação da morfologia matemática, e análise dos componentes conectados. Os resultados são apresentados na Figura 2.

Figura 2. Processamento de nível baixo e médio.

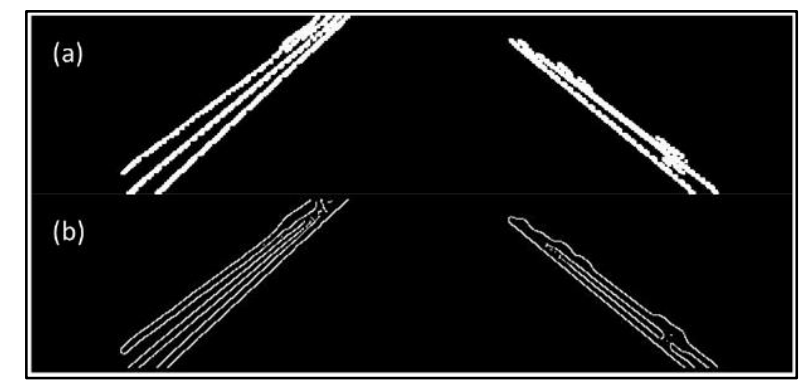

Fonte: (Andrade, 2017).

No processamento de alto nível, a imagem foi dividida em quatro regiões para uma melhor estimativa de faixa quando a pista é curva. Para cada região existe um par de coordenadas para a faixa esquerda e direita, que passa por uma etapa de seleção, priorizando o lado interno da linha. Os autores fizeram o uso da função de interpolação para o agrupamento das regiões. O sistema desenvolvido obteve $96,65 \%$ de acertos. Uma imagem contendo um exemplo de resultado é mostrada na Figura 3.

Figura 3. Reconhecimento de linhas de uma rodovia.

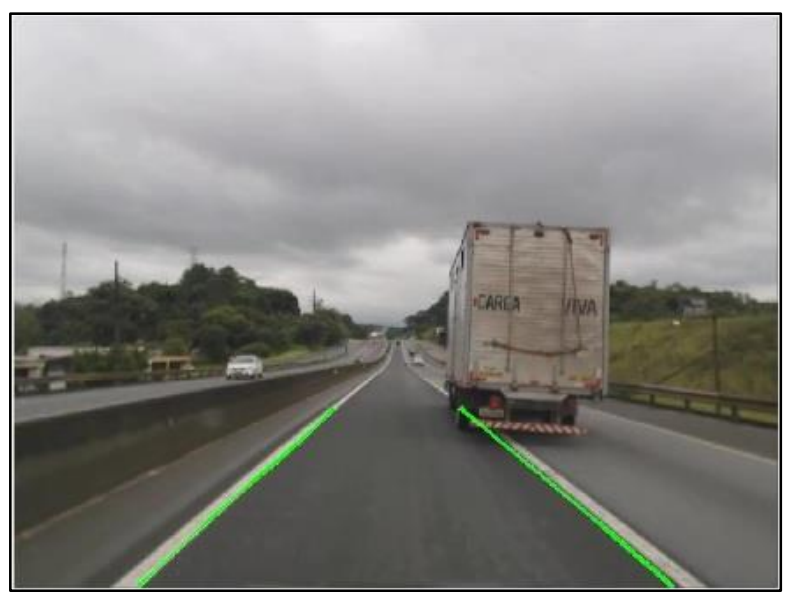

Fonte: (Andrade, 2017). 
Suchitra, Satzoda e Srikanthan (2013) apresentaram um modelo para identificar três tipos de pistas (seccionada, contínua e zigue-zague). É utilizada uma unidade de processamento desenvolvida e denominada Marcação de Pista Básica (BLM - Basic Lane Marking). A identificação da pista é dividida em dois grupos de análise, continuidade espacial e continuidade temporal. A imagem é dividida em quadros que são processados em forma de blocos para encontrar a borda da pista, e após isso, é utilizada a propriedade de continuidade para definir o tipo de pista. A Figura 4 mostra um exemplo de resultado.

Figura 4. Exemplo de identificação de três pistas com faixas diferentes.

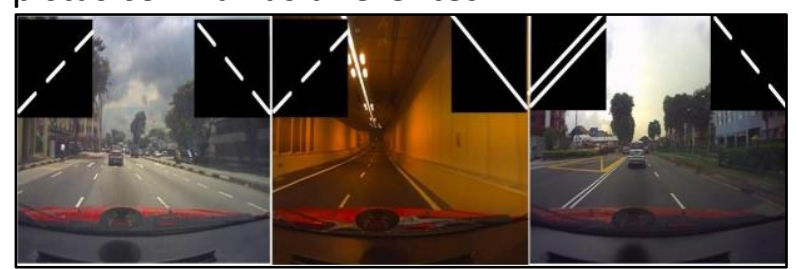

Fonte: (Suchitra, Satzoda e Srikanthan, 2013).

No trabalho de Le et al. (2010) é apresentada uma abordagem utilizando a transformada de Hough para detectar sinais de trânsito que possuem forma circular. Para sinais que possuem outras formas é apresentada a utilização de um detector de bordas para destacar áreas de interesse e possibilitar a utilização de técnicas para extrair informações de formas geométricas, como número de extremidades e ângulos entre duas linhas. Segundo os autores é possível utilizar essa abordagem para aplicações que necessitam de complexidade compatível com tempo real, possibilitando a utilização de câmeras a bordo de um veículo com taxa de reconhecimento de 92,91\%, conforme testes realizados pelos autores.

Lorsakul e Suthakorn (2007) apresentam a utilização de redes neurais para detectar sinais de trânsito. 0 treinamento do sistema foi realizado com imagens de $30 \times 30$ pixels. Assim, para a utilização dessa técnica foi necessário realizar um pré-processamento da imagem para selecionar regiões de interesse baseada na cor e forma da sinalização. A rede neural utilizada no trabalho apresentado foi treinada para reconhecer os sinais de trânsito mais comuns. Segundo os autores, o tempo de processamento é apropriado para aplicações que necessitam de execução em tempo real. As etapas foram projetadas para reduzir a quantidade de processamento, a primeira estratégia consiste em reduzir o número de entradas MLP (Multilayer Perceptron), buscando sempre áreas que realmente existe uma grande possibilidade de ser um sinal e, somente após, realizar a preparação. A segunda estratégia se concentra em buscar uma melhor arquitetura de rede com complexidade reduzida pela seleção de um critério de erro desejável por treinamento. A Figura 5 mostra um exemplo de classificação de uma placa de trânsito de velocidade.

Figura 5. Resultado da classificação.

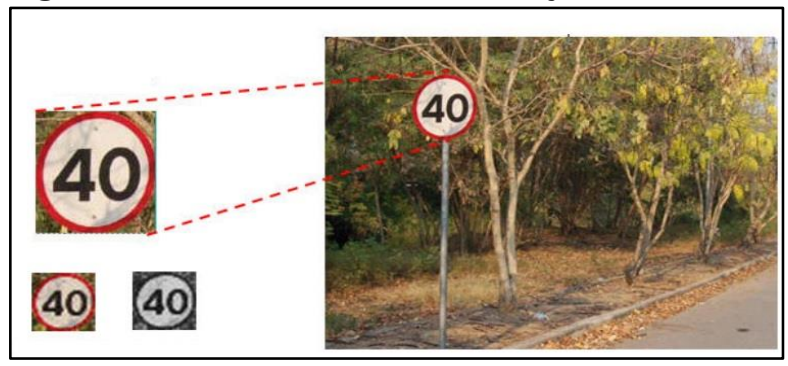

Fonte: (Lorsakul e Suthakorn, 2007).

Hoelscher (2017) propõe dois modelos de Redes Neurais Convolucionais simplificadas, com um número reduzido de camadas, para detectar e classificar placas de trânsito em regiões de interesse extraídas de cenários de trânsito brasileiros e alemães. A rede recebe como entrada um recorte da imagem original para realizar a classificação. O modelo proposto foi treinado para reconhecer seis superclasses conforme ilustrado na Figura 6.

Figura 6. Superclasses de placas de trânsito.

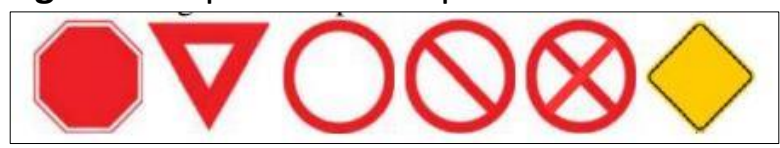

Fonte: (Hoelscher, 2017). 
O modelo proposto combinou duas camadas de convolução (com 32 filtros $5 \times 5 \times 3$ e 128 filtros $3 \times 3$ respectivamente) conectada a uma MLP com duas camadas ocultas de 1024 e 128 neurônios. A camada de saída possui 7 neurônios para as superclasses e 1 para uma classe negativa. A Figura 7 exemplifica o modelo proposto.

Figura 7. Modelo de rede neural convolucional.

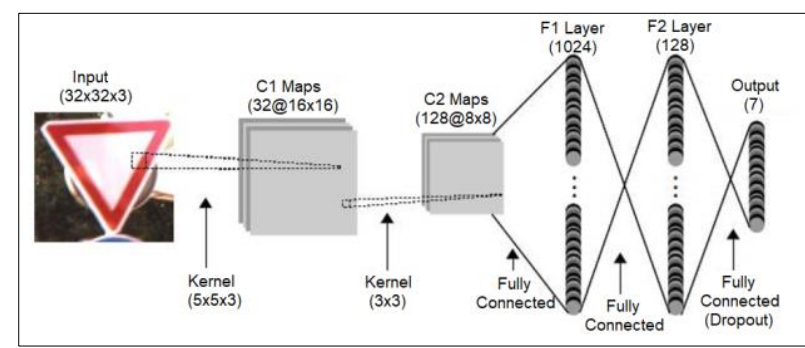

Fonte: (Hoelscher, 2017).

O treinamento foi realizado com 30.000 imagens de sinais de trânsito e alcançou 99,77\% de precisão na classificação.

\section{DESENVOLVIMENTO DO SISTEMA}

\subsection{Visão Geral}

A desatenção é um fator decisivo em questões de segurança no trânsito (ONSV, 2015). Percorrer longas distâncias, distrações durante o percurso e estado emocional do condutor, são exemplos de situações que geram a desatenção no trânsito. Buscando suprir esta desatenção humana, este trabalho visa apresentar informações e emitir alertas em tempo real para o motorista do veículo através de uma solução computacional de baixo custo. Com isso, busca-se auxiliar o motorista para que a direção do veículo possa ser realizada de forma segura e responsável.

Este trabalho apresenta a investigação da utilização de um hardware de baixo custo, sem processador gráfico, para executar a solução computacional de auxílio ao motorista. Essa solução é composta por dois módulos, detecção de faixas de rolamento e reconhecimento de placas de trânsito. Ambos recebem como entrada frames que são obtidos com uma câmera posicionada na parte superior do para-brisa do veículo.

As funções dos módulos são reconhecer a sinalização contida em cada quadro (frame) e realizar o aviso ao motorista. Para a sinalização de linha de pista, sempre é gerado um aviso ao identificar uma invasão em local proibido. Já para as placas de trânsito é informado ao motorista a presença da sinalização na via.

\subsection{Recursos Utilizados}

Para investigar a utilização de um hardware de baixo processamento foi proposto neste trabalho a utilização do Raspberry Pi 3 (Figura 8) com processador quad-core de $1,2 \mathrm{GHz}$ e $1 \mathrm{~GB}$ de memória RAM (Random Access Memory). A placa do Raspberry possui tamanho de $85,60 \mathrm{~mm} \times$ $53,98 \mathrm{~mm}$ e foi projetado para ser de baixo custo.

Figura 8. Raspberry Pi 3.

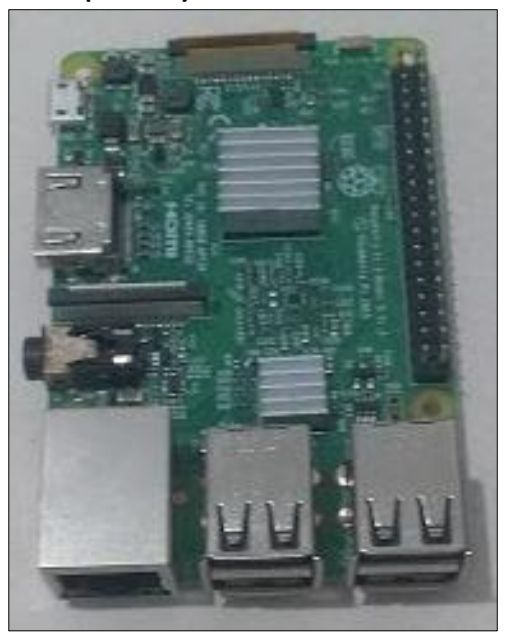

Fonte: (Autor, 2018).

O Raspberry Pi possui uma interface CSI (Camera Interface Serial), que foi utilizada para conectar uma câmera Sunny P5v04a de 5 megapixels (Figura 9). 
Figura 9. Câmera Sunny P5v04a.

Fonte: (Autor, 2018).

A solução foi escrita na linguagem de programação de alto nível Python. Para a implementação dos algoritmos de visão computacional foi utilizada a biblioteca OpenCV (Open Source Computer Vision), que é projetada para eficiência computacional e possui foco em soluções em tempo real. Para o desenvolvimento da rede neural convolucional foi utilizada a API de alto nível Keras em conjunto com o TensorFlow.

\subsection{Métodos}

A solução computacional apresentada neste trabalho segue três etapas para realizar a análise da via. A primeira etapa consiste em obter o frame do vídeo que é capturado pela câmera do Raspberry Pi. Para realizar essa tarefa, a câmera foi posicionada na parte superior do para-brisa do veículo, na parte interna. A imagem da Figura 10 representa um frame capturado de um vídeo gravado no próprio Raspberry Pi.

Figura 10. Frame de vídeo obtido de uma rodovia.

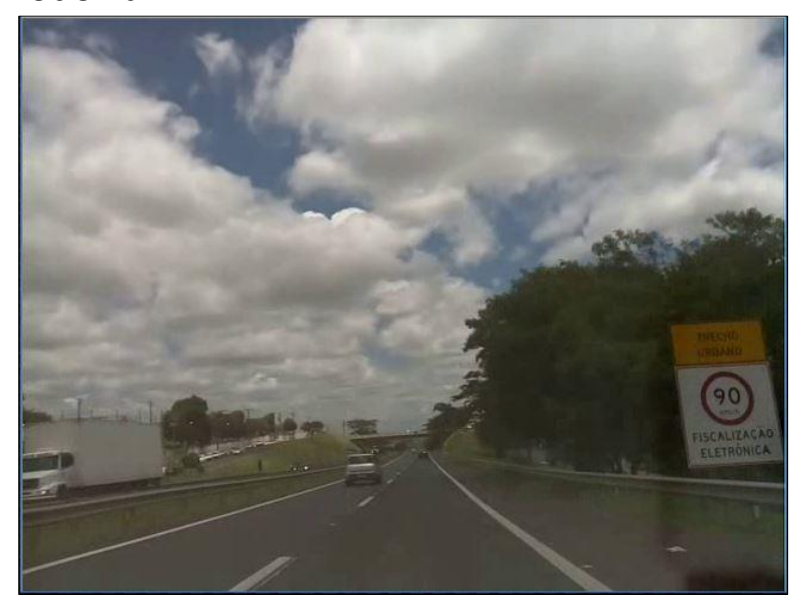

Fonte: (Autor, 2018).
A segunda etapa consiste em processar os frames de vídeo que foram obtidos a partir de alguns vídeos que foram gravados. Muitos frames apresentam ruídos, como exemplo, excesso de luz ou reflexos de objetos que estão no painel do veículo. Desta forma foi necessário preparar a imagem para prosseguir a análise. Um pré-processamento em cada imagem foi realizada para eliminar ruídos e destacar informações relevantes para o reconhecimento dos sinais. O préprocessamento também realiza a seleção da região de interesse para o rastreamento de linhas e para a deteç̧ão e o reconhecimento das placas de velocidade, dê a preferência e pare. Para a região que contêm placas foram selecionadas as laterais esquerda e direita de cada frame. Para as linhas de pista foram selecionadas as regiões abaixo da linha do horizonte, na parte central do frame. Com isso, é possível desconsiderar áreas que possuem a região do céu, paisagens, carros e as laterais da rodovia. Delimitar uma região de interesse proporciona menor custo de processamento e contribui para obter melhor desempenho das etapas seguintes.

O módulo responsável pelo rastreamento das linhas de pista realiza a detecção das bordas na região selecionada. Foi utilizado o algoritmo de Canny (Gonzalez; Woods, 2010) para essa tarefa. Foi necessário definir empiricamente os limiares baixo e alto (50 e 150), em que tais valores possuem grande importância para o reconhecimento de linhas de pista. Valores incorretos podem gerar saídas indesejadas e afetar o processamento das próximas etapas. As modificações desses parâmetros podem aumentar a sensibilidade da detecção das bordas, aumentando o processamento ou impossibilitar o reconhecimento. A Figura 11 mostra um exemplo de resultado de execução algoritmo de Canny com os limiares configurados. 
Figura 11. Região de interesse e aplicação do filtro de Canny.

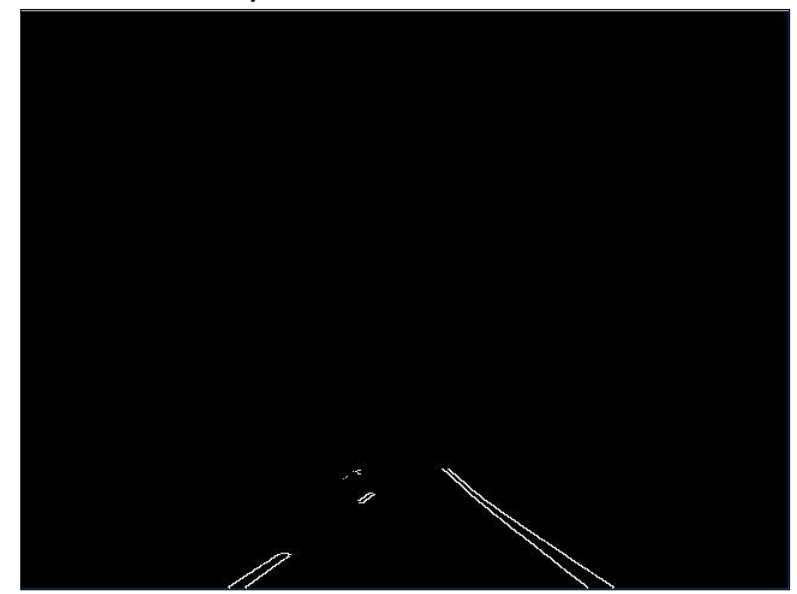

Fonte: (Autor, 2018).

O reconhecimento de linhas é realizado com a Transformada Probabilística de Hough (Gonzalez; Woods, 2010). Esse algoritmo percorre a imagem buscando regiões de bordas que formam retas, e retorna como resposta todos os segmentos encontrados. São obtidos todos os pontos iniciais e finais de cada segmento contido na imagem (frame do vídeo).

Ruídos na imagem podem gerar segmentos de retas que não pertencem às faixas de rolamento. Portanto, esses ruídos, que são segmentos horizontais, foram identificados e eliminados com base no deslocamento vertical da reta (1). Para eliminar um segmento horizontal, deve ser atendida a inequação (2):

$$
\begin{gathered}
\nabla y=y 2-y 1 \\
|\nabla y|<\varepsilon V
\end{gathered}
$$

onde $y 1$ e $y 2$ são as coordenas verticais dos pontos inicial e final que formam o segmento de reta, e $\varepsilon V$ corresponde ao valor mínimo aceitável. Neste trabalho foi definido empiricamente que o deslocamento da reta deve atender ao limiar mínimo $\varepsilon V=5$.

É mantido um histórico do reconhecimento dos últimos 5 frames para auxiliarem na seleção de segmentos de retas que pertencem a faixa de rolamento. Desta forma, é possível eliminar segmentos que diferem muito dos reconhecimentos anteriores. Visto que as linhas tendem a não variar bruscamente de posição. A Figura 12 mostra um resultado após eliminar os segmentos de retas que não pertencem às faixas de rolamento.

Figura 12. Reconhecimento de linhas de faixas de rolamento.

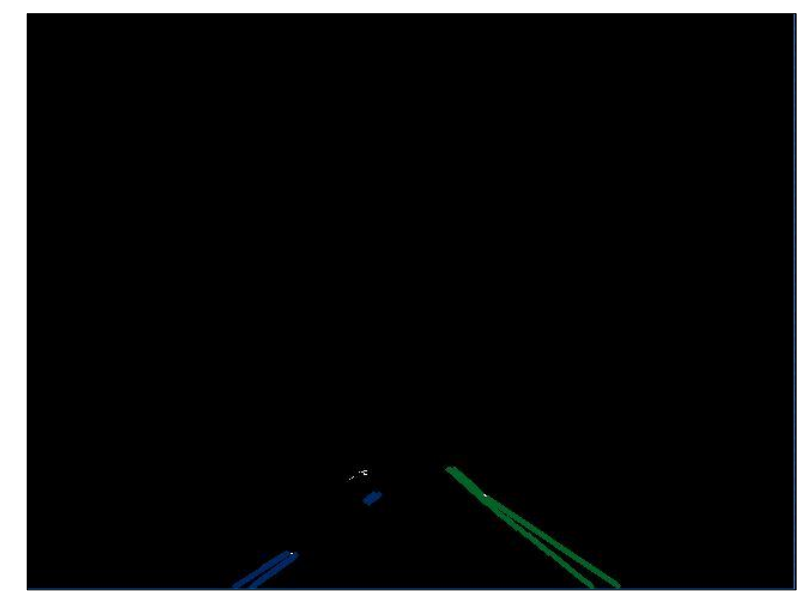

Fonte: (Autor, 2018).

O controle de invasão de pista é realizado conforme o tipo de faixa. Para essa tarefa foi implementado um classificador rápido de linhas, analisando a disposição dos segmentos e seus comprimentos. Foi definido um limiar mínimo de 15 pixels entre dois segmentos. Quando o espaçamento entre retas ultrapassar o limiar, é classificado como seccionada.

A análise da posição do veículo em relação à reta é definida em relação ao centro do frame, pois a câmera deve sempre estar posicionada no centro do para-brisa do veículo. Quando a linha esquerda ou direita se aproxima do centro é realizada a análise do tipo de faixa. No caso de a classificação ser contínua, esta é reconhecida como uma invasão de pista irregular.

O módulo de reconhecimento das placas de sinalização desenvolvido neste trabalho utiliza um modelo de rede neural convolucional inspirado no proposto por Andrade (2017), que pode ser utilizado em dispositivos com baixo poder de processamento. $O$ modelo original combina duas camadas de convolução (com 32 filtros e 128 filtros respectivamente) e operações de max-pooling para reduzir as 
características da imagem, destacando as mais relevantes. A saída da camada de convolução é concatenada e ligada com uma MLP com duas camadas ocultas de 1024 e 128 neurônios. A camada de saída é convertida em probabilidade para cada classe.

No modelo de rede utilizado neste trabalho, que realiza o reconhecimento das placas de trânsito (velocidade, dê a preferência e pare), foi adicionada uma camada convolucional com 128 filtros ao modelo proposto por Andrade (2017).

A base de dados para o treinamento da rede foi criada a partir de imagens que foram obtidas a partir de gravações de vídeos com a câmera do Raspberry Pi embarcada em um veículo. Foram utilizadas técnicas para aumentar a base de dados, como rotações e alterações de brilho. Com isso, o dataset criado possui 1450 imagens para treinamento e 337 para teste. Ao final do treinamento, o modelo alcançou uma precisão de $97,2 \%$ na classificação das placas de sinalizações utilizadas.

\section{EXPERIMENTOS}

Para a realização dos experimentos, um veículo foi equipado com o Raspberry Pi e câmera para realizar gravações de percursos em rodovias na cidade de Presidente Prudente. Dois vídeos foram gravados em dias diferentes e em rodovias distintas. As condições climáticas e de iluminação foram semelhantes para os registros de treino e teste. As gravações dos vídeos foram em dias ensolarados com boas condições de visibilidade. Os tempos médios de processamento de cada frame é apresentado na Tabela 1.
Tabela 1. Tempos de processamento por frame.

\begin{tabular}{c|c}
\hline Tarefa & $\begin{array}{c}\text { Tempo médio em } \\
\text { segundos }\end{array}$ \\
\hline $\begin{array}{c}\text { Reconhecimento } \\
\text { de placas }\end{array}$ & 0.064 \\
\hline $\begin{array}{c}\text { Reconhecimento } \\
\text { de linhas }\end{array}$ & 0.225 \\
\hline
\end{tabular}

Fonte: (Autor, 2018).

Os resultados obtidos com o primeiro vídeo foram satisfatórios. Sendo possível executar a solução em tempo real com taxa de reconhecimento das sinalizações também satisfatórias. A Tabela 2 apresenta os resultados obtidos.

Tabela 2. Resultados do vídeo 1.

\begin{tabular}{c|c|c|c}
\hline Frames & Acertos & Erros & Precisão \\
\hline $\begin{array}{c}\text { Reconhecimento } \\
\text { de placas }\end{array}$ & 3 & 0 & $100 \%$ \\
\hline $\begin{array}{c}\text { Reconhecimento } \\
\text { de linhas }\end{array}$ & 325 & 14 & $95,9 \%$ \\
\hline Saída de pista & 1 & 0 & $100 \%$ \\
\hline
\end{tabular}

Fonte: (Autor, 2018).

A Tabela 3 representa os resultados obtidos como segundo vídeo gravado.

Tabela 3. Resultados do vídeo 2.

\begin{tabular}{c|c|c|c}
\hline Frames & Acertos & Erros & Precisão \\
\hline $\begin{array}{c}\text { Reconhecimento } \\
\text { de placas }\end{array}$ & 12 & 0 & $100 \%$ \\
\hline $\begin{array}{c}\text { Reconhecimento } \\
\text { de linhas }\end{array}$ & 420 & 44 & $90,5 \%$ \\
\hline Saída de pista & 1 & 0 & $100 \%$ \\
\hline
\end{tabular}

Fonte: (Autor, 2018).

A Figura 13 mostra uma saída do sistema com o reconhecimento das sinalizações. 
Figura 13. Reconhecimento das sinalizações em um frame do vídeo 1 utilizado nos testes.

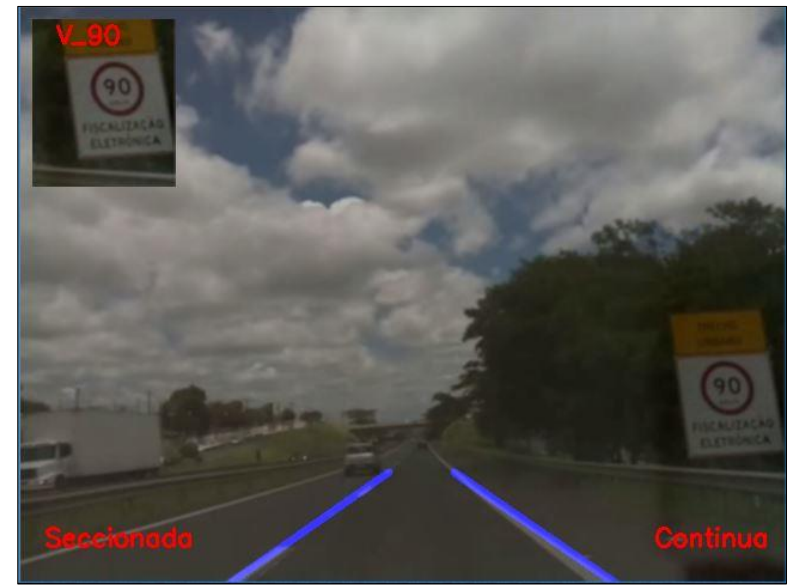

Fonte: (Autor, 2018).

\section{CONSIDERAÇÕES FINAIS}

Neste trabalho foram desenvolvidos algoritmos que realizam a tarefa de auxiliar a percepção humana ao guiar veículos, com execução em hardware de baixo processamento em tempo real. Esse conjunto de algoritmos formam uma solução computacional que possui potencial para viabilizar a implantação em veículos, e com isso reduzir os efeitos causados pela desatenção humana.

Os experimentos mostraram-se satisfatórios em dias ensolarados e em boas condições de visibilidade. O tempo de processamento foi satisfatório para execução em tempo real, mas ainda necessita de melhorias para reduzir o tempo de resposta. Hardwares com processadores especializados para visão computacional e aprendizado de máquina podem ser adicionados ao Raspberry Pi para melhorar o desempenho, como por exemplo, o Intel Movidius.

$O$ dataset de imagens de placas de trânsito utilizado para o treinamento da rede neural convolucional poderia ser ampliado, incluindo adicionar imagens com condições adversas de tempo e luminosidade.

Ampliar as sinalizações reconhecidas e abordar outras infrações de trânsito durante o processo de análise da rodovia, são exemplos de funcionalidades que podem ser adicionadas ao projeto em trabalhos futuros. É de interesse para a segurança dos motoristas e de grande valia, um sistema que além de reconhecer sinalizações, também analise a presença de outros veículos nas vias contrárias ou a existência de obstáculos. Tais análises podem contribuir ainda mais para ampliar as situações de riscos reconhecidas e aumentar o nível de segurança nas rodovias do Brasil.

\section{REFERÊNCIAS}

ANDRADE, D. C. Estratégia para detecção e rastreamento de faixas rodoviárias utilizando uma câmera monocular. 2017. Dissertação (Mestrado em Engenharia Elétrica) - Universidade Tecnológica Federal do Paraná. Ponta Grossa, 2017.

HOELSCHER, I. G. Detecção e classificação de sinalização vertical de trânsito em cenários complexos. 2017. Dissertação (Mestrado em Engenharia Elétrica) - Universidade Federal do Rio Grande do Sul. Porto Alegre, 2017.

CNT - CONFEDERAÇÃO NACIONAL DE TRANSPORTE. Pesquisa rodoviária 2018. Disponível em: http://www.cnt.org.br. Acesso em: 17 dez. 2018.

ONSV. $\mathbf{9 0 \%}$ dos acidentes são causados por falhas humanas, alerta ONSV Disponível em: http://www.onsv.org.br/noticias/90-dosacidentes-sao-causados-por-falhas-humanasalerta-observatorio/. Acesso em: $17 \mathrm{dez}$. 2017.

DANESCU, R.; NEDEVSCHI, S. Detection and classification of painted road objects for intersection assistance applications. In: INTERNATIONAL IEEE CONFERENCE ON INTELLIGENT TRANSPORTATION SYSTEMS (ITSC 2010), 13., Proceedings [...] Funchal, Portugal, 2010, p. 433-438. https://doi.org/10.1109/ITSC.2010.5625261

GONZALEZ, R. C.; WOODS, R. E. Digital Image Processing. 3. ed. São Paulo: Pearson Prentice Hall, 2010. Disponível em: 
https://bv4.digitalpages.com.br/ Acesso em: 17 dez. 2018.

LE, T. T.; TRAN, S. T.; MITA, S.; NGUYEN, T. D. Real Time Traffic Sign Detection Using Color and Shape-Based Features. In: ACIIDS'10 PROCEEDINGS OF THE SECOND INTERNATIONAL CONFERENCE ON INTELLIGENT INFORMATION AND DATABASE SYSTEMS: Part II. Proceeding [...] SpringerVerlag Berlin, Heidelberg, 2010, p 3-9. https://doi.org/10.1007/978-3-642-12101-

$\underline{228}$

LORSAKUL, A.; SUTHAKORN, J. Traffic Sign Recognition for Intelligent Vehicle/Driver Assistance System Using Neural Network on OpenCV. In: INTERNATIONAL CONFERENCE ON UBIQUITOUS ROBOTS AND AMBIENT INTELLIGENCE (URAI 2007), 4., Proceedings... POSTECH, PIRO, KOREA, Nov 22-24, 2007. p. 279-284.

NAN, Z.; WEI, P.; XU, L.; ZHENG, N. Efficient Lane Boundary Detection with SpatialTemporal Knowledge Filtering. Sensors 2016, v. 16, n.8, p. 1276, 2016.https://doi.org/10.3390/s16081276

SUCHITRA, S.; SATZODA, R.; SRIKANTHAN, T. Identifying lane types: A modular approach. In: INTERNATIONAL IEEE CONFERENCE ON INTELLIGENT TRANSPORTATION SYSTEMS (ITSC 2013), 16., Proceedings [...] The Hague, Netherlands, 2013, p. 1929-1934. https://doi.org/10.1109/ITSC.2013.6728511

VISVIKIS, C.; SMITH, T. L.; PITCHER, M.; SMITH, R, Study on lane departure warning and lane change assistant systems. (PPR 374). Wokingham, UK: Transport Research Laboratory, 2008. 